\title{
Autorité et sanctions en enseignement
}

\section{HRONIQUE • Éthique en éducation}

Au début du siècle dernier, des pédagogues rêvent d'une école sans autorité ni sanctions. À cet égard, Célestin Freinet plaide pour une discipline sans punition.Il propose de fonder une école communautaire pour les enfants d'ouvriers. John Dewey défend à son tour des idées similaires (2011). Une école sans pratique disciplinaire ni sanction n'est-elle qu'une utopie pédagogique?

Les expériences libertaires de l'école de Summerhill fondée par Alexander Sutherland Neill en 1921 et de l'école de Hambourg ouverte dans les années 1920 n'ont pas été concluantes. L'école de Hambourg représente la forme la plus radicale du refus de l'autorité et des règles pour encadrer les élèves. Les enseignants de cette école pensaient que l'absence de contraintes pouvait favoriser l'autonomie des élèves et la réussite scolaire. Léchec de Hambourg montre les limites d'une école libertaire.

Eirick Prairat écrit à cet égard que l'expérience de Hambourg est un " échec d'autant plus amer que pendant plus de dix ans ces maîtres novateurs ont fait preuve d'un enthousiasme et d'une énergie peu communs. Zeidler, un des inspirateurs du projet, dut reconnaître non sans tristesse, après plusieurs années d'expérience que "partout où l'on se laisse guider par une confiance sans bornes dans le tact des enfants, dans leur force de volonté, dans leur persévérance, dans la sûreté de leur instinct et dans la tolérance des individus à former une communauté... on voit se former des bandes d'indisciplinés" ». (Prairat, 2003, p. 50). 
Les élèves soustraits aux règles et à la vigilance de l'autorité des enseignants risquent la régression dans une sauvagerie primitive. Dans Sa Majesté des Mouches, William Golding met en scène des écoliers britanniques, naufragés sur une île déserte, sacrifiant l'un d'entre eux par plaisir. Libérées des pressions morales des adultes, les pulsions sanguinaires des enfants n'ont plus de frein. Leur cruauté est sans limites. William Golding tenait à illustrer dans son roman que l'éducation qu'ont reçue les enfants avant d'être abandonnés sur une île est impuissante à réguler définitivement leur potentiel de violence. En fait, la violence fait retour dès la disparition d'une figure d'autorité qui a le mandat d'administrer les règles disciplinaires et de faire pression pour qu'elles soient respectées.

Le relâchement de la discipline est devenu l'un des plus grands problèmes des systèmes scolaires occidentaux. Il est vrai qu'avant les années 1960, les instituteurs et institutrices exerçaient une autorité répressive, parfois tyrannique, sur des élèves sensés obéissants. Leur autorité, légitimée par les instances religieuses et civiles, était définie comme un pouvoir de commander et de se faire obéir. Or, cette autorité qui abusait de la menace, de l'humiliation et du châtiment corporel n'est plus acceptable ni pratiquée de nos jours. Les mœurs pédagogiques de l'école contemporaine se sont, pour le bonheur de tous, modernisées.

Toutefois, la fin de l'autorité traditionnelle ne fait pas disparaître l'autorité comme le pensait Jean Houssaye (1996). L'autorité scolaire s'exerce autrement, assurément avec plus d'humanité. Du moins, les enseignants cherchent à l'exercer autrement, le plus souvent en mettant de l'avant sa visée préventive et protectrice plutôt que sa visée répressive et punitive. Ils n'y arrivent pas toujours, d'où les immenses difficultés que rencontrent nombre d'enseignants, novices et expérimentés, dans leur tâche de gestion des troubles de comportement des élèves.

L'autorité des enseignants est en crise. Hannah Arendt (1972) avait prévu cette crise dès les années 1950. Elle est constatée par les spécialistes de l'éducation après la mouvementée époque de la contre-culture. Or, les élèves ne peuvent pas être laissés à eux-mêmes, sans les pressions d'un adulte en autorité. Il y a donc lieu de repenser une autorité en phase avec une époque qui cherche à respecter les droits des enfants (Renaut, 2004).

Dans les termes de Max Weber, l'autorité est un pouvoir légitime. Quelles sont ses sources de légitimité? Dans l'école traditionnelle, les institutions politiques et religieuses permettent aux enseignants - mais aussi aux parents - d'exercer sur les enfants une violence dite éducative. Les paroles humiliantes, les menaces de coups et les châtiments corporels sont alors acceptables. L'autorité des adultes est perçue comme un pouvoir naturel sur les enfants.

Dans les mœurs de l'époque, il était naturel que les élèves obéissent aux enseignants, comme à la maison il paraissait naturel que les enfants obéissent à leur parent. Le moindre écart de discipline d'un élève était puni par le maître d'école, puis par le père à la maison.

Ce temps où l'autorité du maître d'école paraissait naturelle est révolu. L'usage de la force physique, des menaces et des paroles humiliantes ne fait plus partie de la panoplie des interventions pédagogiques acceptables. Dorénavant, l'enseignant ne fait plus autorité parce qu'il est craint des élèves, mais parce qu'il est respecté. Mais comment arrive-t-il à être respecté? 
Les enseignants doivent dorénavant compter sur les élèves pour prétendre assumer une fonction d'autorité. Lorsqu'ils se présentent à leurs élèves en début d'année scolaire ou dans le cadre d'un remplacement, leur autorité n'est pas immédiatement reconnue par les élèves. Ils ne peuvent plus mettre en scène leur autorité en faisant valoir un pouvoir menaçant et brutal sur eux. Les élèves n'acceptent plus la logique de domination des maîtres. Leur autorité doit donc être conquise, reconnue et consentie par les élèves qui peuvent l'accepter, la rejeter ou la fragiliser.

L'enseignant contemporain fait reconnaître sa légitimité en s'engageant auprès des élèves à respecter les normes éthiques les plus élevées de sa profession. Il communique aux élèves que son jugement sera guidé par des valeurs de respect, de justice, de responsabilité, de clémence, de modération et de bienveillance. Il doit assurer les élèves qu'il agira en tout temps avec professionnalisme. Cela signifie que son autorité sera régie par des normes connues des élèves.

L'adhésion à des normes éthiques transforme l'administration des règles scolaires et des sanctions disciplinaires. Lorsqu'il émettait une sanction, l'enseignant d'autrefois faisait valoir son pouvoir de punir, de se faire obéir, en fait de soumettre les élèves à son autorité. Dorénavant, les enseignants font valoir l'autorité des règles écrites pour tous. Des règles qui indiquent les bons comportements et les sanctions. Les élèves obéissent aux règles plutôt qu'à la personne de l'enseignant. Dans cette perspective, une sanction devient la réponse connue et prévisible au manquement à une règle scolaire. Les enseignants n'ont plus à inventer des sanctions selon les situations d'indiscipline. Leur rôle, comme représentants des règles, vise plutôt à les rappeler et à les appliquer.

La sanction ne vise plus comme autrefois l'obéissance des élèves, et encore moins à les soumettre au pouvoir des enseignants. Elle fait partie d'une logique hiérarchique d'interventions disciplinaire. Elle est utilisée en dernier recours, après les avertissements, les réprimandes, la perte de points de comportement, etc., ou lorsque la transgression à la règle est très grave. La sanction vise à faire cesser immédiatement un comportement inacceptable selon les règles, et non selon l'humeur de l'enseignant. Ces derniers ne peuvent s'en servir pour satisfaire leurs propres intérêts, car ils doivent toujours agir dans l'intérêt des élèves. Elle est pédagogique dans la mesure où elle vise à responsabiliser l'élève. La justice de la sanction tient à la cohérence d'un enseignant dans ses interventions.

L'intérêt d'une telle position est de deux ordres : d'une part, protéger les élèves contre l'arbitraire des sanctions perçues par eux comme injustes; d'autre part, protéger les enseignants contre les représailles des élèves.

Une sanction n'est pas une fin en soi. Elle est une intervention, parmi une panoplie d'autres interventions, qui rappelle l'obligation du respect des règles établies pour le bien de tous. Une sanction n'est pas un acte de représailles ni un acte qui vise à soumettre les élèves à l'autorité des enseignants. L'élève n’obéit pas aux enseignants, mais obéit à des règles « établies » que les enseignants administrent avec circonspection étant donné leur adhésion à des normes éthiques communes. La sanction en classe, en fait, peut être administrée à la manière des sanctions sportives. Un joueur fautif sera sanctionné par un arbitre selon des règles connues. C'est parce que les règles et les sanctions sont connues par les joueurs qu'elles leur sont acceptables. 
Rappelons en terminant qu'on ne peut faire reposer sur les épaules de chaque enseignant le défi de faire reconnaître son autorité. C'est pourquoi leur autorité doit être légitimée par l'adhésion à des normes éthiques communes à tous les enseignants. Les élèves doivent savoir que tous les enseignants méritent le même respect de leur autorité du fait qu'ils s'engagent à respecter des normes professionnelles.

Quelle que soit la méthode utilisée pour soutenir et encourager les élèves à respecter les règles de la classe, il y aura toujours des problèmes de comportement. Un élève peut comprendre pourquoi une règle est nécessaire. Mais cela ne le retient pas nécessairement de transgresser les règles. Or, lorsqu'une règle est transgressée, l'élève connaît à l'avance la sanction.

Il est erroné de penser que les élèves nont pas besoin d'un dispositif de contrôle pour se comporter dans le respect des règles scolaires. Un système disciplinaire est nécessaire pour assurer le bon ordre dans une école comme dans la classe. Loin d'être un constat d'échec de la relation pédagogique, un système de discipline dans la classe lui est conditionnel. Comme l'ont montré Gauthier et Tardif (2004), la discipline fait partie intégrante de la pédagogie.

\section{Références}

Arendt, H. (1972). La crise de la culture : huit exercices de pensée politique. Paris : Gallimard.

Dewey, J. (2011). Démocratie et éducation. Suivi de Expérience et éducation. Paris : Armand Colin.

Gauthier, C. et Tardif, M. (dir.) (2004). La pédagogie. Théories et pratiques de l'Antiquité à nos jours (2éd.). Montréal, QC : Gaëtan Morin.

Golding, W. (1983). Sa Majesté des Mouches. Paris : Gallimard.

Houssaye, J. (1996). Autorité ou éducation? Paris : ESF.

Neill, A. S. (1985). Libres enfants de Summerbill. Paris : La Découverte.

Prairat, E. (2003). La sanction en éducation. Paris : Presses universitaires de France.

Renaut, A. (2004). La fin de l'autorité. Paris : Flammarion.

\section{Pour citer cet article}

Jeffrey, D. (2018). Autorité et sanctions en enseignement. Formation et profession, 26(2), 99-102. http://dx.doi.org/10.18162/fp.2018.a145 